\title{
Exponentiated Exponential Lomax Distribution and its Properties
}

\author{
Maradesa Adeleke ${ }^{1 *}$, Akomolafe Abayomi Ayodele ${ }^{2}$ and Adebola Femi Barnabas ${ }^{3}$ \\ ${ }^{1,2,3}$ Department of Statistics, Federal University of Technology Akure, Nigeria
}

\begin{abstract}
This research study the generalization of exponentiated version of Exponential lomax Distribution (ELD) called Exponentiated exponential lomax distribution (EEPD) through its distribution function and mathematical derivation of their moment, reliability, cumulative distribution function, Renyi Entropy and hazard rate function, Median, Quartile and Quantile Function. The distribution was found to generalize some known distributions thereby providing a great flexibility in modeling heavy tailed, skewed and bimodal distributions.
\end{abstract}

Keywords: Exponentiated-exponential Lomax Distribution (EELD), Moment generating function, Hazard Function, Entropy, Median, Quartile, Quantile Function.

DOI: $10.7176 / \mathrm{MTM} / 9-1-01$

\section{Overview of the research}

Many researchers have worked on aspect of compounding two or more probability distributions to obtain family of hybrid distributions which are more efficient than their parent distributions due to addition of more parameters which increase the flexibility of the mixture of distributions in tracking many random phenomena which cannot be easily modeled by their parent distributions. Many authors have also worked on compounding beta distribution with other distributions. The beta family of distribution became popular some years back, which include beta-normal (Eugene \&Famoye, 2002); beta-Gumbel (Nadarajah \& Kotz, 2004), beta-Weibull (Famoye, Lee\& Olugbenga, 2005), Beta-exponential (Nadarajah \&Kotz, 2006); bata-Rayleigh (Akinsete \&Lowe, 2009); beta-Laplace (Kozubowski Nadarajah, 2008) beta-pareto (Akinete, Faye \& Lee, 200), Barreto-Souza, Santos, and Cordeiro (2009) constructed Beta generalized exponential, Beta-half-Cauchy was presented by Cordeiro, and Lemonte (2011), Gastellares, Montenegero, and Gauss derived Beta Log-normal , while Morais ,Cordeiro ,and Audrey (2011) introduced Beta Generalized Logistic, the Beta Burr III Model for Lifetime Data, Betahyperbolic Secant(BHS) by Mattheas, David(2007), Beta Fre'chet by Nadarajah, and Gupta(2004), betahalfnormal, Akomolafe AA and Maradesa A (2017), beta-Gamma, beta-f, beta-t, beta-beta, beta-modified weibull, beta-nakagami among others. Some articles also evolved regarding exponential-pareto by Kareema Abdul Al-Kadim and Mohammed Abdulhussain (2013).Transmuted pareto, meronvci f. and Puka L. (2014), transmuted lomax, Ashour S.K and Eltehiwy M.A (2013), Exponential lomax, El-bassiouny et al (2015) and transmuted frechet , Mahmoud M.R and Mandouh R.M (2013). It is in this view that this research is structured to propose new hybrid distributions with a view to studying its properties and application to real life data to reflect the flexibility, stability and consistency of this hybrid model as compared to its parent distributions.

2 Derivation of Exponentiated Exponential Lomax Distribution (EEPD) 
The cdf of the exponentiated family of distribution according to Nadarajah and Kotz is defined as (1) below:

$=F(x ; \tau, \theta)=\left(1-(1-G(x ; \tau))^{\theta}, \theta>0\right.$

To obtain the pdf $f(x ; \tau, \alpha)$, obtain the derivative of (1)

$$
\begin{aligned}
& f(x ; \tau, \alpha) \quad=\quad \frac{d}{d x}\left[\left(1-(1-G(x ; \tau))^{\theta}\right)\right] \\
& \left.\frac{d}{d x}(1)-\frac{d}{d x}(1-G(x ; \tau))^{\theta}\right)=-g \theta(x ; \tau) .-(1-G(x ; \tau))^{\theta-1} \\
& =f(x ; \tau, \alpha)=\theta g(x ; \tau)(1-G(x ; \tau))^{\theta-1}
\end{aligned}
$$

Where

\section{$g(x ; \tau), G(x ; \tau)$ and $\tau$ are $p d f, c d f$ and parameter vector of parent distribution}

The cdf of Exponential lomax is defined according to El-bassiouny et al (2015) is given by (3).

$=F(x ; \beta, \lambda, \theta)=1-e^{\left.-\lambda\left(\frac{\beta}{\mathrm{x}+\beta}\right)\right)^{-\alpha}}$

$=f(x ; \beta, \lambda, \theta)=\frac{d}{d x}(1)-\frac{d}{d x}\left(e^{-\lambda\left(\frac{\beta}{\mathrm{x}+\beta}\right)^{-\alpha}}\right)=\frac{\lambda \alpha}{\beta}\left(\frac{\beta}{\mathrm{x}+\beta}\right)^{-\alpha+1} e^{-\lambda\left(\frac{\beta}{\mathrm{x}+\beta}\right)^{-\alpha}}$

Where (3) and (4) are the cdf and pdf of exponential El-bassiouny et al (2015).

Now

let $g(x ; \tau)=\frac{\lambda \alpha}{\beta}\left(\frac{\beta}{\mathrm{x}+\beta}\right)^{-\alpha+1} e^{-\lambda\left(\frac{\beta}{\mathrm{x}+\beta}\right)^{-\alpha}}$ and $G(x ; \tau)=1-e^{-\lambda\left(\frac{\beta}{\mathrm{x}+\beta}\right)^{-\alpha}}$ where $\tau=(\beta, \lambda, \alpha)$

Substitute for $G(x ; \tau)=1-e^{-\lambda\left(\frac{\beta}{x+\beta}\right)^{-\alpha}}$ in (1) to obtain the cdf of Exponentiated exponential pareto distribution (EELD).

$$
\begin{aligned}
= & F_{E E L D}(x ; \beta, \lambda, \theta, \alpha)=\left(1-(1-G(x ; \tau))^{\theta}, \theta>0\right. \\
& F_{E E L D}(x ; \beta, \lambda, \theta, \alpha)=\left(1-\left[\left(1-\left(1-e^{-\lambda\left(\frac{\beta}{\mathrm{x}+\beta}\right)^{-\alpha}}\right)\right)^{\theta}\right], \alpha>0, \theta>0, \beta>0, \lambda>0 \text { and } x>\right. \\
= & 0
\end{aligned}
$$

The cumulative density function is represented by (5), the Exponentiated exponential lomax (EELD) has an increased number of parameter which can increase its flexibility.

By using equation (2) the pdf of EEPD can be obtained as (6) when substituting for $g(x ; \tau)=\frac{\lambda \alpha}{\beta}\left(\frac{\beta}{\mathrm{x}+\beta}\right)^{-\alpha+1} e^{-\lambda\left(\frac{\beta}{\mathrm{x}+\beta}\right)^{-\alpha}}$ and $G(x ; \tau)=1-e^{-\lambda\left(\frac{\beta}{\mathrm{x}+\beta}\right)^{-\alpha}}$ where $\tau=(\beta, \lambda, \alpha)$ 


$$
f_{(E E L D)}(x ; \beta, \lambda, \theta, \alpha)=\theta g(x ; \tau)(1-G(x ; \tau))^{\theta-1}
$$

$=$

$\frac{\lambda \theta \alpha}{\beta}\left(\frac{\beta}{\mathrm{x}+\beta}\right)^{-\alpha+1} e^{-\lambda\left(\frac{\beta}{\mathrm{x}+\beta}\right)^{-\alpha}}\left(1-\left(1-e^{\left.-\lambda\left(\frac{\beta}{\mathrm{x}+\beta}\right)\right)^{-\alpha}}\right)\right)^{\theta-1} \quad \alpha>0, \theta>0, \beta>0, \lambda>0$ and $x>$

The equation (6) is the pdf of EEPD. The pdf of EELD has the unique property of reducing to the pdf of ELD when $\theta=1$

\subsection{Reliability}

$$
\begin{aligned}
& =R(x)=1-F(x ; \beta, \lambda, \theta, \alpha)=1-\left[1-\left(1-\left(1-e^{-\lambda\left(\frac{\beta}{\mathrm{x}+\beta}\right)^{-\alpha}}\right)\right)^{\theta}\right] \\
& =\left(1-\left(1-e^{-\lambda\left(\frac{\beta}{\mathrm{x}+\beta}\right)^{-\alpha}}\right)\right)^{\theta}=\left(e^{-\lambda\left(\frac{\beta}{\mathrm{x}+\beta}\right)^{-\alpha}}\right)^{\theta}
\end{aligned}
$$

\subsection{Hazard Rate Function}

$$
\begin{aligned}
& H(x)=\frac{f(x)}{R(x)}=\frac{\frac{\lambda \theta \alpha}{\beta}\left(\frac{\beta}{\mathrm{x}+\beta}\right)^{-\alpha+1} e^{-\lambda\left(\frac{\beta}{\mathrm{x}+\beta}\right)^{-\alpha}}\left(1-\left(1-e^{-\lambda\left(\frac{\beta}{\mathrm{x}+\beta}\right)^{-\alpha}}\right)\right)^{\theta-1}}{\left(e^{-\lambda\left(\frac{\beta}{\mathrm{x}+\beta}\right)^{-\alpha}}\right)^{\theta}} \\
& =\frac{\lambda \theta \alpha}{\beta}\left(\frac{\beta}{\mathrm{x}+\beta}\right)^{-\alpha+1} e^{-\lambda\left(\frac{\beta}{\mathrm{x}+\beta}\right)^{-\alpha}}\left(1-\left(1-e^{-\lambda\left(\frac{\beta}{\mathrm{x}+\beta}\right)^{-\alpha}}\right)\right)^{\theta-1} \cdot\left(e^{-\lambda\left(\frac{\beta}{\mathrm{x}+\beta}\right)^{-\alpha}}\right)^{-\theta} \\
& \mathrm{H}(\mathrm{x})=\frac{\lambda \theta \alpha}{\beta}\left(\frac{\beta}{\mathrm{x}+\beta}\right)^{-\alpha+1}\left(e^{-\lambda\left(\frac{\beta}{\mathrm{x}+\beta}\right)^{-\alpha}}\right)^{1-\theta}\left(1-\left(1-e^{-\lambda\left(\frac{\beta}{\mathrm{x}+\beta}\right)^{-\alpha}}\right)\right)^{\theta-1} \\
& \mathrm{H}(\mathrm{x})=\frac{\lambda \theta \alpha}{\beta}\left(\frac{\beta}{\mathrm{x}+\beta}\right)^{-\alpha+1}\left(e^{-\lambda\left(\frac{\beta}{\mathrm{x}+\beta}\right)^{-\alpha}}\right)^{1-\theta} \cdot\left(e^{-\lambda\left(\frac{\beta}{\mathrm{x}+\beta}\right)^{-\alpha}}\right)^{\theta-1} \\
& \mathrm{H}(\mathrm{x})=\frac{\lambda \theta \alpha}{\beta}\left(\frac{\beta}{\mathrm{x}+\beta}\right)^{-\alpha+1}
\end{aligned}
$$

\subsection{Odd Function}

$O(x)=\frac{F(x ; \alpha, \beta, \theta, \lambda)}{R(x)}=\frac{\left(1-\left(1-\left(1-e^{-\lambda\left(\frac{\beta}{\mathrm{x}+\beta}\right)^{-\alpha}}\right)^{\theta}\right)\right.}{\left(e^{-\lambda\left(\frac{\beta}{\mathrm{x}+\beta}\right)^{-\alpha}}\right)^{\theta}}=\frac{\left(1-\left(e^{-\lambda\left(\frac{\beta}{\mathrm{x}+\beta}\right)^{-\alpha}}\right)^{\theta}\right)}{\left(e^{-\lambda\left(\frac{\beta}{\mathrm{x}+\beta}\right)^{-\alpha}}\right)^{\theta}}$ 
$=\left(1-\left(e^{-\lambda\left(\frac{\beta}{x+\beta}\right)^{-\alpha}}\right)^{\theta}\right) \cdot\left(e^{-\lambda\left(\frac{\beta}{x+\beta}\right)^{-\alpha}}\right)^{-\theta}$

\subsection{Moment Generating Function}

$$
\begin{aligned}
& =M_{x}(t)=E e^{t x}=\int_{0}^{\infty} e^{t x} f(x ; \tau) d x=\sum_{r}^{\infty} \frac{t^{r}}{r !} \int_{0}^{\infty} x^{r} f(x ; \tau) d x=\sum_{r}^{\infty} \frac{t^{r}}{r !} E x^{r} \\
& =E x^{r}=\int_{0}^{\infty} x^{r} \frac{\lambda \theta \alpha}{\beta}\left(\frac{\beta}{\mathrm{x}+\beta}\right)^{-\alpha+1} e^{-\lambda\left(\frac{\beta}{\mathrm{x}+\beta}\right)^{-\alpha}}\left(1-\left(1-e^{-\lambda\left(\frac{\beta}{\mathrm{x}+\beta}\right)^{-\alpha}}\right)\right)^{\theta-1} d x \\
& =\int_{0}^{\infty} x^{r} \frac{\lambda \theta \alpha}{\beta}\left(\frac{\beta}{\mathrm{x}+\beta}\right)^{-\alpha+1} e^{-\lambda\left(\frac{\beta}{\mathrm{x}+\beta}\right)^{-\alpha}} \cdot\left(e^{-\lambda\left(\frac{\beta}{\mathrm{x}+\beta}\right)^{-\alpha}}\right)^{\theta-1} \mathrm{dx} \\
& =\int_{0}^{\infty} x^{r} \frac{\lambda \theta \alpha}{\beta}\left(\frac{\beta}{\mathrm{x}+\beta}\right)^{-\alpha+1} e^{-\lambda\left(\frac{\beta}{\mathrm{x}+\beta}\right)^{-\alpha}} \cdot e^{-\lambda(\theta-1)\left(\frac{\beta}{\mathrm{x}+\beta}\right)^{-\alpha}} d x \\
& =\int_{0}^{\infty} x^{r} \frac{\lambda \theta \alpha}{\beta}\left(\frac{\beta}{\mathrm{x}+\beta}\right)^{-\alpha+1} e^{(-\lambda-\theta \lambda+\lambda)\left(\frac{\beta}{\mathrm{x}+\beta}\right)^{-\alpha}} d x=\int_{0}^{\infty} x^{r} \frac{\lambda \theta \alpha}{\beta}\left(\frac{\beta}{\mathrm{x}+\beta}\right)^{-\alpha+1} e^{-\theta \lambda\left(\frac{\beta}{\mathrm{x}+\beta}\right)^{-\alpha}} d x
\end{aligned}
$$

We can make use of the relations below and make necessary substitution in (10)

$$
\begin{aligned}
& \mathrm{u}=\theta \lambda\left(\frac{\beta}{\mathrm{x}+\beta}\right)^{-\alpha} ; \frac{u}{\theta \lambda}=\left(\frac{\beta}{\mathrm{x}+\beta}\right)^{-\alpha} ;\left(\frac{\beta}{\mathrm{x}+\beta}\right)^{\alpha}=\frac{\theta \lambda}{u} ; \frac{\beta}{\mathrm{x}+\beta}=\frac{(\theta \lambda)^{\frac{1}{\alpha}}}{\mathrm{u}^{\frac{1}{\alpha}}} ; \beta u^{\frac{1}{\alpha}}=(\theta \lambda)^{\frac{1}{\alpha}}(x+\beta) \\
& \mathrm{x}=\frac{\beta u^{\frac{1}{\alpha}}}{(\theta \lambda)^{\frac{1}{\alpha}}}-\beta ; \frac{d x}{d u}=\frac{\beta u^{\frac{1}{\bar{\alpha}}-1}}{\alpha(\theta \lambda)^{\frac{1}{\alpha}}} \cdot \text { substitute for } \mathrm{dx}, \mathrm{x} \text { and } \mathrm{u} \text { in (10). } \\
& =\int_{0}^{\infty} x^{r} \frac{\lambda \theta \alpha}{\beta}\left(\frac{\beta}{\mathrm{x}+\beta}\right)^{-\alpha+1} e^{-\mathrm{u}} \cdot \frac{\beta u^{\frac{1}{\alpha}-1}}{\alpha(\theta \lambda)^{\frac{1}{\alpha}}} d u=\frac{\lambda \theta}{(\lambda \theta)^{\frac{1}{\alpha}}} \int_{0}^{\infty} x^{r}\left(\frac{\beta}{\frac{\beta u^{\frac{1}{\alpha}}}{(\theta \lambda)^{\frac{1}{\alpha}}}-\beta+\beta}\right)^{-\alpha+1} e^{-\mathrm{u}} \cdot u^{\frac{1}{\alpha}-1} d u \\
& =\frac{\lambda \theta}{(\lambda \theta)^{\frac{1}{\alpha}}} \int_{0}^{\infty} x^{r}\left(\frac{\beta(\theta \lambda)^{\frac{1}{\alpha}}}{\beta(u)^{\frac{1}{\alpha}}}\right)^{-\alpha+1} \cdot e^{-\mathrm{u}} \cdot u^{\frac{1}{\alpha}-1} d u=\frac{\lambda \theta}{(\lambda \theta)^{\frac{1}{\alpha}}} \int_{0}^{\infty} x^{r} \cdot \frac{(\theta \lambda)^{\frac{1}{\alpha}(-\alpha+1)}}{u^{\frac{1}{\alpha^{(}(-\alpha+1)}}} e^{-\mathrm{u}} \cdot u^{\frac{1}{\alpha}-1} d u \\
& =\frac{\lambda \theta}{(\lambda \theta)^{\frac{1}{\alpha}}} \int_{0}^{\infty} x^{r} \cdot \frac{(\theta \lambda)^{-1+\frac{1}{\alpha}}}{u^{-1+\frac{1}{\alpha}}} e^{-\mathrm{u}} \cdot u^{\frac{1}{\alpha}-1} d u=\frac{(\lambda \theta) \cdot(\theta \lambda)^{-1+\frac{1}{\alpha}}}{(\lambda \theta)^{\frac{1}{\alpha}}} \int_{0}^{\infty} x^{r} \cdot \frac{1}{u^{-1+\frac{1}{\alpha}}} e^{-\mathrm{u}} \cdot u^{\frac{1}{\alpha}-1} d u \\
& =(\lambda \theta)^{1-\frac{1}{\alpha}} \cdot(\theta \lambda)^{-1+\frac{1}{\alpha}} \int_{0}^{\infty} x^{r} \cdot u^{-\left(-1+\frac{1}{\alpha}\right)} e^{-\mathrm{u}} \cdot u^{\frac{1}{\alpha}-1} d u \\
& =\int_{0}^{\infty} x^{r} \cdot u^{-\left(-1+\frac{1}{\alpha}\right)} \cdot u^{\frac{1}{\alpha}-1} e^{-\mathrm{u}} d u=\int_{0}^{\infty} x^{r} e^{-\mathrm{u}} d u \\
& \text { Now put for } \mathrm{x}=\frac{\beta u^{\frac{1}{\alpha}}}{(\theta \lambda)^{\frac{1}{\alpha}}}-\beta \text { in (14) }
\end{aligned}
$$


$=\int_{0}^{\infty}\left(\frac{\beta u^{\frac{1}{\alpha}}}{(\theta \lambda)^{\frac{1}{\alpha}}}-\beta\right)^{r} e^{-\mathrm{u}} d u=\sum_{j=0}^{r}\left(\begin{array}{l}r \\ j\end{array}\right)\left(\frac{\beta}{\sqrt[\alpha]{\theta \lambda}}\right)^{j}(-\beta)^{r-j} \int_{0}^{\infty} u \frac{j}{\alpha} e^{-\mathrm{u}} d u$

$=E x^{r}=\sum_{j=0}^{r}\left(\begin{array}{l}r \\ j\end{array}\right)\left(\frac{\beta}{\sqrt[\alpha]{\theta \lambda}}\right)^{j}(-\beta)^{r-j} \Gamma\left(\frac{j}{\alpha}+1\right), \mathrm{r}=1,2,3 \ldots$

Substitute for (16) in (7) to obtain the moment generating function of Exponentiated Exponential Lomax (EELD).

$=\sum_{r}^{\infty} \frac{t^{r}}{r !} \sum_{j=0}^{r}\left(\begin{array}{l}r \\ j\end{array}\right)\left(\frac{\beta}{\sqrt[\alpha]{\theta \lambda}}\right)^{j}(-\beta)^{r-j} \Gamma\left(\frac{j}{\alpha}+1\right) \mathrm{r}=1,2,3$

Let $W_{r}=\sum_{r}^{\infty} \frac{t^{r}}{r !}$, then (17) can be re-written as (18)

$=M_{x}(t)=W_{r} \sum_{j=0}^{r}\left(\begin{array}{l}r \\ j\end{array}\right)\left(\frac{\beta}{\sqrt[\alpha]{\theta \lambda}}\right)^{j}(-\beta)^{r-j} \Gamma\left(\frac{j}{\alpha}+1\right)$

The equation (18) is the moment generating function of Exponential exponentiated lomax distribution (EELD) and it has many relations to the moment generating function of Exponential lomax distribution (ELD). This is because the mgf of EELD reduces to $\mathrm{mgf}$ of ELD when the parameter $\theta=1$. It means the addition of another parameter $\theta$ made EELD four parameters-hybrid-distribution and the additional parameter gives it flexibility over ELD in modeling data with skewed distribution.

\subsection{Renyi Entropy}

The measure of the uncertain Situation for the random variable $\mathrm{X}$ with $\mathrm{X} \sim \operatorname{EELD}(\lambda, \alpha, \beta, \theta)$ can be calculated by the Renyi entropy (19) which can be expressed by the following relation.

$=\delta_{r}=\frac{1}{1-r} \log \left(\int_{0}^{\infty} f^{r}(x) d x\right)$

\subsubsection{Proposition 1}

If $\mathrm{X}$ is a random variable that is distributed as $\operatorname{EELD}(\mathrm{X} \sim \operatorname{EELD}(\lambda, \alpha, \beta, \theta))$, then the measure of uncertain situation for the random variable $\mathrm{X}$ is given as: $\delta_{r}=\frac{1}{1-r} \log \left(\frac{\alpha \lambda \theta}{\beta}\right)^{r} \frac{\beta}{\alpha}(\lambda \theta r)^{-r+\frac{r}{\alpha}-\frac{1}{\alpha}} \Gamma\left(\frac{(\alpha-1)(\mathrm{r}-1)}{\alpha}+1\right)$

\section{Proof}

$$
\begin{aligned}
&= \frac{1}{1-r} \log \int_{0}^{\infty}\left(\frac{\lambda \theta \alpha}{\beta}\left(\frac{\beta}{\mathrm{x}+\beta}\right)^{-\alpha+1} e^{-\theta \lambda\left(\frac{\beta}{\mathrm{x}+\beta}\right)^{-\alpha}}\right)^{r} d x \\
& \frac{1}{1-r} \cdot\left(\frac{\alpha \lambda \theta}{\beta}\right)^{r} \log \int_{0}^{\infty}\left(\frac{\beta}{\mathrm{x}+\beta}\right)^{\mathrm{r}(-\alpha+1)} e^{-\theta \lambda \mathrm{r}\left(\frac{\beta}{\mathrm{x}+\beta}\right)^{-\alpha}} d x(20)
\end{aligned}
$$




$$
\begin{aligned}
& \mathrm{y}=\theta \lambda \mathrm{r}\left(\frac{\beta}{\mathrm{x}+\beta}\right)^{-\alpha} ; \frac{y}{\theta \lambda \mathrm{r}}=\left(\frac{\beta}{\mathrm{x}+\beta}\right)^{-\alpha} ;\left(\frac{\beta}{\mathrm{x}+\beta}\right)^{\alpha}=\frac{\theta \lambda \mathrm{r}}{y} ; \frac{\beta}{\mathrm{x}+\beta}=\frac{(\theta \lambda \mathrm{r})^{\frac{1}{\alpha}}}{\mathrm{y}^{\frac{1}{\alpha}}} ; \beta y^{\frac{1}{\alpha}}=(\theta \lambda \mathrm{r})^{\frac{1}{\alpha}}(x+\beta) \\
& \mathrm{x}=\frac{\beta y^{\frac{1}{\alpha}}}{(\theta \lambda \mathrm{r})^{\frac{1}{\alpha}}}-\beta ; \frac{d x}{d y}=\frac{\beta y^{\frac{1}{\alpha}-1}}{\alpha(\theta \lambda \mathrm{r})^{\frac{1}{\alpha}}}, \text { substitute for } \mathrm{y}, \mathrm{dx}, \text { and } \mathrm{x} \text { in (20) }
\end{aligned}
$$

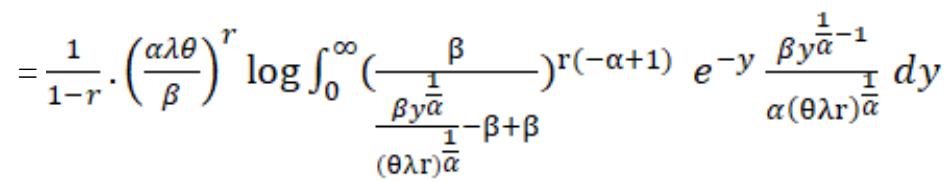

$$
\begin{aligned}
& =\frac{1}{1-r} \cdot \log \left(\frac{\alpha \lambda \theta}{\beta}\right)^{r} \cdot \frac{\beta}{\alpha(\theta \lambda \mathrm{r})^{\frac{1}{\alpha}}} \int_{0}^{\infty}\left(\frac{(\theta \lambda \mathrm{r})^{\frac{1}{\alpha}}}{y^{\frac{1}{\alpha}}}\right)^{\mathrm{r}(-\alpha+1)} \cdot e^{-y} y^{\frac{1}{\alpha}-1} d y \\
& =\frac{1}{1-r} \cdot \log \left(\frac{\alpha \lambda \theta}{\beta}\right)^{r} \cdot \frac{\beta}{\alpha(\theta \lambda \mathrm{r})^{\frac{1}{\alpha}}} \int_{0}^{\infty} \frac{(\theta \lambda \mathrm{r})^{\frac{r}{\alpha}(-\alpha+1)}}{y^{\frac{r}{\alpha}(-\alpha+1)}} e^{-y} y^{\frac{1}{\alpha}-1} d y \\
& =\frac{1}{1-r} \cdot \log \left(\frac{\alpha \lambda \theta}{\beta}\right)^{r} \cdot \frac{\beta}{\alpha(\theta \lambda \mathrm{r})^{\frac{1}{\alpha}}} \cdot(\theta \lambda \mathrm{r})^{\frac{r}{\alpha}(-\alpha+1)} \int_{0}^{\infty} \frac{1}{y^{\frac{r}{\alpha}(-\alpha+1)}} e^{-y} y^{\frac{1}{\alpha}-1} d y \\
& =\frac{1}{1-r} \cdot \log \left(\frac{\alpha \lambda \theta}{\beta}\right)^{r} \cdot \frac{\beta}{\alpha(\theta \lambda \mathrm{r})^{\frac{1}{\alpha}}} \cdot(\theta \lambda \mathrm{r})^{\frac{r}{\alpha}(-\alpha+1)} \int_{0}^{\infty} y^{-\frac{r}{\alpha}(-\alpha+1)} e^{-y} y^{\frac{1}{\alpha}-1} d y \\
& =\frac{1}{1-r} \cdot \log \left(\frac{\alpha \lambda \theta}{\beta}\right)^{r} \cdot \frac{\beta}{\alpha} \cdot(\theta \lambda \mathrm{r})^{-\frac{1}{\alpha}}(\theta \lambda \mathrm{r})^{\frac{r}{\alpha}(-\alpha+1)} \int_{0}^{\infty} y^{r-\frac{r}{\alpha}+\frac{1}{\alpha}-1} e^{-y} d y \\
& =\frac{1}{1-r} \cdot \log \left(\frac{\alpha \lambda \theta}{\beta}\right)^{r} \cdot \frac{\beta}{\alpha} \cdot(\theta \lambda \mathrm{r})^{-\frac{1}{\alpha}}(\theta \lambda \mathrm{r})^{\frac{r}{\alpha}(-\alpha+1)} \cdot M \\
& \mathrm{M}=\int_{0}^{\infty} y^{r-\frac{r}{\alpha}+\frac{1}{\alpha}-1} e^{-y} d y \\
& \mathrm{u}=y^{-\frac{\mathrm{r}}{\alpha}+\mathrm{r}+\frac{1}{\alpha}} ; \mathrm{dy}=\frac{\mathrm{y}^{\frac{\mathrm{r}}{\alpha}-\mathrm{r}-\frac{1}{\alpha}+1}}{-\frac{\mathrm{r}}{\alpha}+\mathrm{r}+\frac{1}{\alpha}} d u \text { and we can used } \mathrm{y}=u^{\frac{1}{-\frac{\mathrm{r}}{\alpha}+\mathrm{r}+\frac{1}{\alpha}}} \\
& =-\frac{1}{\frac{r}{\alpha}-\mathrm{r}-\frac{1}{\alpha}} \int_{0}^{\infty} e^{-u^{-\frac{1}{\alpha}+\mathrm{r}+\frac{1}{\alpha}}} d u . \\
& \mathrm{T}=\int_{0}^{\infty} e^{-u^{\frac{1}{-\frac{\mathrm{r}}{\alpha}+\mathrm{r}+\frac{1}{\alpha}}}} d u
\end{aligned}
$$

Equation (28)is a special integral of Incomplete gamma function

$$
\mathrm{T}=-\left(-\frac{\mathrm{r}}{\alpha}+\mathrm{r}+\frac{1}{\alpha}\right) \Gamma\left(-\frac{\mathrm{r}}{\alpha}+\mathrm{r}+\frac{1}{\alpha}, u^{\frac{1}{-\frac{\mathrm{r}}{\alpha}+\mathrm{r}+\frac{1}{\alpha}}}\right)
$$


Substitute for $\mathrm{T}$ in (27) to obtain (29)

$=-\frac{1}{\frac{\mathrm{r}}{\alpha}-\mathrm{r}-\frac{1}{\alpha}} \cdot\left(-\frac{\mathrm{r}}{\alpha}+\mathrm{r}+\frac{1}{\alpha}\right) \Gamma\left(-\frac{\mathrm{r}}{\alpha}+\mathrm{r}+\frac{1}{\alpha}, u^{\frac{1}{-\frac{\mathrm{r}}{\alpha}+\mathrm{r}+\frac{1}{\alpha}}}\right)$

Recall that $\mathrm{y}=u^{\frac{1}{-\frac{\mathrm{r}}{\alpha}+\mathrm{r}+\frac{1}{\alpha}}}$, then

$=\frac{1}{\frac{\mathrm{r}}{\alpha}-\mathrm{r}-\frac{1}{\alpha}} \cdot\left(-\frac{\mathrm{r}}{\alpha}+\mathrm{r}+\frac{1}{\alpha}\right) \Gamma\left(-\frac{\mathrm{r}}{\alpha}+\mathrm{r}+\frac{1}{\alpha}, y\right)=-\Gamma\left(\frac{(\alpha-1) r+1}{\alpha}, y\right)$

Equation (30) can be rewritten as - $\Gamma\left(\frac{(\alpha-1)(r-1)}{\alpha}+1, y\right)$

Therefore $M=\Gamma\left(\frac{(\alpha-1)(r-1)}{\alpha}+1\right)$. We can substitute for $M$ in $\quad(26)$ to obtain the proposed renyi entropy for EEPD.

$=\frac{1}{1-r} \cdot \log \left(\frac{\alpha \lambda \theta}{\beta}\right)^{r} \cdot \frac{\beta}{\alpha} \cdot(\theta \lambda \mathrm{r})^{-\frac{1}{\alpha}}(\theta \lambda \mathrm{r})^{\frac{r}{\alpha}}(-\alpha+1) \Gamma\left(\frac{(\alpha-1)(r-1)}{\alpha}+1\right)$

$\delta_{r}=\frac{1}{1-r} \cdot \log \left(\frac{\alpha \lambda \theta}{\beta}\right)^{r} \cdot \frac{\beta}{\alpha} \cdot(\theta \lambda \mathrm{r})^{-r+\frac{r}{\alpha}-\frac{1}{\alpha}} \Gamma\left(\frac{(\alpha-1)(r-1)}{\alpha}+1\right)$

The (31) above is the proposed renyi entropy for EELD.

\section{$2.6 \beta$-Entropy}

$\boldsymbol{\beta}$ is defined as one parameter generalization of the Shannon entropy. $\boldsymbol{\beta}$-entropy can be defined as:

$=H_{\bar{\beta}}=\frac{1}{\bar{\beta}-1}\left[1-\int_{0}^{\infty} f^{\bar{\beta}}(x) d x\right] \quad$ for $\bar{\beta} \neq 1$

\subsubsection{Proposition 2}

Let $\mathrm{X}$ be a random variable that is distributed as $\mathrm{X} \sim \operatorname{EELD}(\alpha, \beta, \theta, \lambda)$, then its one parameter generalization of the Shannon entropy ( $\boldsymbol{\beta}$-entropy) is defined as:

$H_{\bar{\beta}}=\frac{\beta}{\overline{(\beta}-1) \alpha}\left(\frac{\lambda \theta \alpha}{\beta}\right)^{\bar{\beta}}(\theta \lambda \bar{\beta})^{-\bar{\beta}+\frac{\bar{\beta}}{\alpha}-\frac{1}{\alpha}} \Gamma\left(\frac{(\alpha-1)(\bar{\beta}-1)}{\alpha}+1\right)$

\section{Proof}




$$
\begin{aligned}
& =H_{\bar{\beta}}=\frac{1}{\bar{\beta}-1} \int_{0}^{\infty}\left(\frac{\lambda \theta \alpha}{\beta}\left(\frac{\beta}{\mathrm{x}+\beta}\right)^{-\alpha+1} e^{-\theta \lambda\left(\frac{\beta}{\mathrm{x}+\beta}\right)^{-\alpha}}\right)^{\bar{\beta}} d x \\
& \frac{1}{\bar{\beta}-1} \cdot\left(\frac{\lambda \theta \alpha}{\beta}\right)^{\bar{\beta}} \int_{0}^{\infty}\left(\frac{\beta}{\mathrm{x}+\beta}\right)^{\bar{\beta}(-\alpha+1)} e^{-\theta \lambda \bar{\beta}\left(\frac{\beta}{\mathrm{x}+\beta}\right)^{-\alpha}} d x(33) \\
& \mathrm{y}=\theta \lambda \bar{\beta}\left(\frac{\beta}{\mathrm{x}+\beta}\right)^{-\alpha} ; \frac{y}{\theta \lambda \bar{\beta}}=\left(\frac{\beta}{\mathrm{x}+\beta}\right)^{-\alpha} ;\left(\frac{\beta}{\mathrm{x}+\beta}\right)^{\alpha}=\frac{\theta \lambda \bar{\beta}}{y} ; \frac{\beta}{\mathrm{x}+\beta}=\frac{(\theta \lambda \bar{\beta})^{\frac{1}{\alpha}}}{\mathrm{y}^{\frac{1}{\alpha}}} ; \beta y^{\frac{1}{\alpha}}=(\theta \lambda \bar{\beta})^{\frac{1}{\alpha}}(x+\beta) \\
& \mathrm{x}=\frac{\beta y^{\frac{1}{\alpha}}}{(\theta \lambda \bar{\beta})^{\frac{1}{\alpha}}}-\beta ; \frac{d x}{d y}=\frac{\beta y^{\frac{1}{\alpha}-1}}{\alpha(\theta \lambda \bar{\beta})^{\frac{1}{\alpha}}}, \text { substitute for } \mathrm{y}, \mathrm{dx}, \text { and } \mathrm{x} \text { in (33) } \\
& \left.=\frac{1}{\bar{\beta}-1}\left(\frac{\lambda \theta \alpha}{\beta}\right)^{\bar{\beta}} \int_{0}^{\infty} \frac{\left(\frac{\beta}{\beta y^{\frac{1}{\alpha}}}-\beta+\beta\right.}{(\theta \lambda \bar{\beta})^{\frac{1}{\alpha}}}\right)^{\bar{\beta}(-\alpha+1)} e^{-y} \frac{\beta y^{\frac{1}{\alpha}-1}}{\alpha(\theta \lambda \bar{\beta})^{\frac{1}{\alpha}}} d y \\
& =\frac{1}{\bar{\beta}-1}\left(\frac{\lambda \theta \alpha}{\beta}\right)^{\bar{\beta}} \frac{\beta}{\alpha(\theta \lambda \bar{\beta})^{\frac{1}{\alpha}}} \int_{0}^{\infty}\left(\frac{(\theta \lambda \bar{\beta})^{\frac{1}{\alpha}}}{y^{\frac{1}{\alpha}}}\right)^{\bar{\beta}(-\alpha+1)} . e^{-y} y^{\frac{1}{\alpha}-1} d y \\
& =\frac{1}{\bar{\beta}-1}\left(\frac{\lambda \theta \alpha}{\beta}\right)^{\bar{\beta}}(\theta \lambda \bar{\beta})^{\frac{\bar{\beta}}{\alpha}(-\alpha+1)} \frac{\beta}{\alpha(\theta \lambda \bar{\beta})^{\frac{1}{\alpha}}} \int_{0}^{\infty} \frac{1}{y^{\frac{\beta}{\alpha}(-\alpha+1)}} e^{-y} y^{\frac{1}{\alpha}-1} d y \\
& =\frac{1}{\bar{\beta}-1}\left(\frac{\lambda \theta \alpha}{\beta}\right)^{\bar{\beta}}(\theta \lambda \bar{\beta})^{\frac{\bar{\beta}}{\alpha}(-\alpha+1)} \frac{\beta}{\alpha(\theta \lambda \bar{\beta})^{\frac{1}{\alpha}}} \int_{0}^{\infty} y^{-\frac{r}{\alpha}(-\alpha+1)} e^{-y} y^{\frac{1}{\alpha}-1} d y \\
& =\frac{1}{\bar{\beta}-1}\left(\frac{\lambda \theta \alpha}{\beta}\right)^{\bar{\beta}} \frac{\beta}{\alpha}(\theta \lambda \bar{\beta})^{\frac{\bar{\beta}}{\alpha}(-\alpha+1)} \cdot(\theta \lambda \bar{\beta})^{-\frac{1}{\alpha}} \int_{0}^{\infty} y^{-\frac{r}{\alpha}(-\alpha+1)} e^{-y} y^{\frac{1}{\alpha}-1} d y \\
& H_{\bar{\beta}}=\frac{1}{\bar{\beta}-1}\left(\frac{\lambda \theta \alpha}{\beta}\right)^{\bar{\beta}} \frac{\beta}{\alpha}(\theta \lambda \bar{\beta})^{-\bar{\beta}+\frac{\bar{\beta}}{\alpha}-\frac{1}{\alpha}} \Gamma\left(\frac{(\alpha-1)(\bar{\beta}-1)}{\alpha}+1\right) \\
& H_{\bar{\beta}}=\frac{\beta}{\overline{(\beta}-1) \alpha}\left(\frac{\lambda \theta \alpha}{\beta}\right)^{\bar{\beta}}(\theta \lambda \bar{\beta})^{-\bar{\beta}+\frac{\bar{\beta}}{\alpha}-\frac{1}{\alpha}} \Gamma\left(\frac{(\alpha-1)(\bar{\beta}-1)}{\alpha}+1\right)
\end{aligned}
$$

Therefore the $\beta$-entropy is given in equation (40)

2.7 Median

$0.5=\int_{0}^{\infty} f(x ; \tau) d x=1-\left(1-\left(1-e^{-\lambda\left(\frac{\beta}{m+\beta}\right)^{-\alpha}}\right)^{\theta}\right.$ 
$-0.5=-\left(1-\left(1-e^{-\lambda\left(\frac{\beta}{m+\beta}\right)^{-\alpha}}\right)^{\theta}=0.5=\left(e^{-\lambda\left(\frac{\beta}{m+\beta}\right)^{-\alpha}}\right)^{\theta}\right.$

$0.5=e^{-\lambda \theta\left(\frac{\beta}{\mathrm{m}+\beta}\right)^{-\alpha}}$, therefore

$\ln 0.5=-\lambda \theta\left(\frac{\beta}{\mathrm{m}+\beta}\right)^{-\alpha}=-0.693=-\lambda \theta\left(\frac{\beta}{\mathrm{m}+\beta}\right)^{-\alpha}$

$=0.693=\lambda \theta\left(\frac{\beta}{\mathrm{m}+\beta}\right)^{-\alpha}$, then $\frac{0.693}{\lambda \theta}=\frac{(\mathrm{m}+\beta)^{\alpha}}{\beta^{\alpha}}$

$=\beta^{\alpha} \cdot \frac{0.693}{\lambda \theta}=(\mathrm{m}+\beta)^{\alpha}$, which can be simplify as $: \mathrm{m}+\beta=\beta \cdot\left(\frac{0.693}{\lambda \theta}\right)^{\frac{1}{\alpha}}$

$=\mathrm{m}=\beta \sqrt[\alpha]{\frac{0.693}{\lambda \theta}}-\beta$

Therefore the median of EEPD is $\mathrm{m}=\beta\left(\sqrt[\alpha]{\frac{0.693}{\lambda \theta}}-1\right)$

\subsection{Quartiles}

\subsubsection{First Quartile}

$0.25=\int_{0}^{q 1} f(x ; \tau) d x=1-\left(1-\left(1-e^{-\lambda\left(\frac{\beta}{\mathrm{q} 1+\beta}\right)^{-\alpha}}\right)^{\theta}\right.$

$-0.75=-\left(1-\left(1-e^{-\lambda\left(\frac{\beta}{\mathrm{q} 1+\beta}\right)^{-\alpha}}\right)^{\theta}=0.75=\left(e^{-\lambda\left(\frac{\beta}{\mathrm{q} 1+\beta}\right)^{-\alpha}}\right)^{\theta}\right.$

$0.75=e^{-\lambda \theta\left(\frac{\beta}{\mathrm{q} 1+\beta}\right)^{-\alpha}}$, therefore

$\ln 0.75=-\lambda \theta\left(\frac{\beta}{\mathrm{q} 1+\beta}\right)^{-\alpha}=-0.2877=-\lambda \theta\left(\frac{\beta}{\mathrm{q} 1+\beta}\right)^{-\alpha}$

$=0.2877=\lambda \theta\left(\frac{\beta}{\mathrm{q} 1+\beta}\right)^{-\alpha}$, then $\frac{0.693}{\lambda \theta}=\frac{(\mathrm{q} 1+\beta)^{\alpha}}{\beta^{\alpha}}$

$=\beta^{\alpha} \cdot \frac{0.2877}{\lambda \theta}=(\mathrm{q} 1+\beta)^{\alpha}$, which can be simplify as $: \mathrm{q} 1+\beta=\beta \cdot\left(\frac{0.693}{\lambda \theta}\right)^{\frac{1}{\alpha}}$

$=\mathrm{q} 1=\beta \sqrt[\alpha]{\frac{0.693}{\lambda \theta}}-\beta$

Therefore the first quartile of EEPD is $\mathrm{q} 1=\beta\left(\sqrt[\alpha]{\frac{0.2877}{\lambda \theta}}-1\right)$ 


\subsubsection{Third Quartile}

$0.75=\int_{0}^{q 3} f(x ; \tau) d x=1-\left(1-\left(1-e^{-\lambda\left(\frac{\beta}{q_{3}+\beta}\right)^{-\alpha}}\right)^{\theta}\right.$

$-0.25=-\left(1-\left(1-e^{-\lambda\left(\frac{\beta}{q 3+\beta}\right)^{-\alpha}}\right)^{\theta}=0.25=\left(e^{-\lambda\left(\frac{\beta}{q 3+\beta}\right)^{-\alpha}}\right)^{\theta}\right.$

$0.25=e^{-\lambda \theta\left(\frac{\beta}{q 3+\beta}\right)^{-\alpha}}$, therefore

$\ln 0.25=-\lambda \theta\left(\frac{\beta}{\mathrm{q} 3+\beta}\right)^{-\alpha}=-0.1 .3863=-\lambda \theta\left(\frac{\beta}{\mathrm{q} 3+\beta}\right)^{-\alpha}$

$=1.3863=\lambda \theta\left(\frac{\beta}{\mathrm{q} 3+\beta}\right)^{-\alpha}$, then $\frac{1.3863}{\lambda \theta}=\frac{(\mathrm{q} 3+\beta)^{\alpha}}{\beta^{\alpha}}$

$=\beta^{\alpha} \times \frac{1.3863}{\lambda \theta}=(\mathrm{q} 3+\beta)^{\alpha}$, which can be simplify as $: \mathrm{q} 3+\beta=\beta \cdot\left(\frac{1.3863}{\lambda \theta}\right)^{\frac{1}{\alpha}}$

$=\mathrm{q} 3=\beta \sqrt[\alpha]{\frac{1.3863}{\lambda \theta}}-\beta$

Therefore the first quartile of EEPD is $\mathrm{q} 3=\beta\left(\sqrt[\alpha]{\frac{1.3863}{\lambda \theta}}-1\right)$

\subsection{Quantile Function}

$F(x)=1-\left(1-\left(1-e^{-\lambda\left(\frac{\beta}{\mathrm{x}+\beta}\right)^{-\alpha}}\right)^{\theta}\right.$

If we let $\mathrm{u}=\mathrm{F}(\mathrm{x})$, then $u=1-\left(1-\left(1-e^{-\lambda\left(\frac{\beta}{\mathrm{x}+\beta}\right)^{-\alpha}}\right)^{\theta}\right.$

$$
\begin{aligned}
& \mathrm{u}-1=-\left(1-\left(1-e^{-\lambda\left(\frac{\beta}{\mathrm{x}+\beta}\right)^{-\alpha}}\right)^{\theta}=1-u=\left(1-\left(1-e^{-\lambda\left(\frac{\beta}{\mathrm{x}+\beta}\right)^{-\alpha}}\right)^{\theta}\right.\right. \\
& 1-\mathrm{u}=\left(e^{-\lambda\left(\frac{\beta}{\mathrm{x}+\beta}\right)^{-\alpha}}\right)^{\theta}=1-u=e^{-\lambda \theta\left(\frac{\beta}{\mathrm{x}+\beta}\right)^{-\alpha}} \\
& \ln (1-\mathrm{u})=-\lambda \theta\left(\frac{\beta}{\mathrm{x}+\beta}\right)^{-\alpha} \\
& =-\ln (1-\mathrm{u})=\lambda \theta\left(\frac{\beta}{\mathrm{x}+\beta}\right)^{-\alpha}=\ln (1-\mathrm{u})^{-1}=\lambda \theta\left(\frac{\beta}{\mathrm{x}+\beta}\right)^{-\alpha}=\ln \frac{1}{(1-\mathrm{u})}=\lambda \theta\left(\frac{\beta}{\mathrm{x}+\beta}\right)^{-\alpha} \\
& =\ln \frac{1}{(1-\mathrm{u})}=\frac{\lambda \theta(\mathrm{x}+\beta)^{\alpha}}{\beta^{\alpha}}=\frac{\beta^{\alpha}}{\lambda \theta} \cdot \ln \frac{1}{(1-\mathrm{u})}=(\mathrm{x}+\beta)^{\alpha}=\frac{\beta}{(\lambda \theta)^{\frac{1}{\alpha}}}\left(\ln \frac{1}{(1-\mathrm{u})}\right)^{\frac{1}{\alpha}}=\mathrm{x}+\beta
\end{aligned}
$$


$\mathrm{x}=\sqrt[\alpha]{\ln \frac{1}{(1-\mathrm{u})} \cdot \frac{1}{\lambda \theta}} \beta-\beta=\beta\left(\sqrt[\alpha]{\ln \frac{1}{(1-\mathrm{u})} \cdot \frac{1}{\lambda \theta}}-1\right)$

Therefore the quantile function of EELD is represented by $\mathrm{x}=\beta\left(\alpha \sqrt{\ln \frac{1}{(1-\mathrm{u})} \cdot \frac{1}{\lambda \theta}}-1\right)$

\section{Maximum Likelihood Estimation (MLE)}

$\operatorname{Lf}(\mathrm{x} ; \alpha, \beta, \theta, \lambda)=\prod_{i=1}^{n} \frac{\lambda \theta \alpha}{\beta}\left(\frac{\beta}{\mathrm{x}_{\mathrm{i}}+\beta}\right)^{-\alpha+1} e^{-\lambda\left(\frac{\beta}{\mathrm{x}_{\mathrm{i}}+\beta}\right)^{-\alpha}}\left(1-\left(1-e^{-\lambda\left(\frac{\beta}{\mathrm{x}_{\mathrm{i}}+\beta}\right)^{-\alpha}}\right)\right)^{\theta-1}$

$\operatorname{Lf}(\mathrm{x} ; \alpha, \beta, \theta, \lambda)=\prod_{i=1}^{n} \frac{\lambda \theta \alpha}{\beta}\left(\frac{\beta}{\mathrm{x}_{\mathrm{i}}+\beta}\right)^{-\alpha+1} e^{-\theta \lambda\left(\frac{\beta}{\mathrm{x}_{\mathrm{i}}+\beta}\right)^{-\alpha}}$

$\ln \operatorname{Lf}(\mathrm{x} ; \alpha, \beta, \theta, \lambda)=\operatorname{nln} \alpha+n \ln \lambda+\operatorname{nln} \theta-n \ln \beta+(-\alpha+1) \sum_{i=1}^{n} \ln \left(\frac{\beta}{\mathrm{x}_{\mathrm{i}}+\beta}\right)-\lambda \theta \sum_{i=1}^{n}\left(\frac{\beta}{\mathrm{x}_{\mathrm{i}}+\beta}\right)^{-\alpha}$

Now, obtain the partial derivative of (43) with respect to each parameter

$$
\begin{aligned}
& =\frac{\partial \ln L f(x ; \alpha, \beta, \theta, \lambda)}{\partial \lambda}=\frac{n}{\lambda}-\theta \sum_{i=1}^{n}\left(\frac{\beta}{x_{i}+\beta}\right)^{-\alpha}=0 \\
& =\frac{\partial \ln L f(x ; \alpha, \beta, \theta, \lambda)}{\partial \theta}=\frac{n}{\theta}-\lambda \sum_{i=1}^{n}\left(\frac{\beta}{x_{i}+\beta}\right)^{-\alpha}=0 \\
& =\frac{\partial \ln L f(x ; \alpha, \beta, \theta, \lambda)}{\partial \alpha}=\frac{n}{\alpha}-\sum_{i=1}^{n} \ln \left(\frac{\beta}{x_{i}+\beta}\right)+\lambda \theta \sum_{i=1}^{n}\left(\frac{\beta}{x_{i}+\beta}\right)^{-\alpha} \ln \left(\frac{\beta}{x_{i}+\beta}\right)=0 \\
& =\frac{\partial \ln L f(x ; \alpha, \beta, \theta, \lambda)}{\partial \beta}=\frac{-n}{\beta}+(-\alpha+1) \frac{\partial}{\partial \beta} \sum_{i=1}^{n} \ln \left(\frac{\beta}{x_{i}+\beta}\right)-\lambda \theta \frac{\partial}{\partial \beta} \sum_{i=1}^{n}\left(\frac{\beta}{x_{i}+\beta}\right)^{-\alpha}=0
\end{aligned}
$$

We can obtain the estimate of the parameter through numerical method, specifically Newton Raphson Algorithm.

From (44), $\frac{n}{\lambda}-\theta \sum_{i=1}^{n}\left(\frac{\beta}{x_{i}+\beta}\right)^{-\alpha}=0 ; \frac{n}{\lambda}=\theta \sum_{i=1}^{n}\left(\frac{\beta}{x_{1}+\beta}\right)^{-\alpha}$

$=\hat{\lambda}=\frac{n}{\theta \sum_{i=1}^{n}\left(\frac{\beta}{x_{1}+\beta}\right)^{-a}}$ and $\widehat{\theta}$ can be obtained from (45) as $\hat{\theta}=\frac{n}{\lambda \sum_{i=1}^{n}\left(\frac{\beta}{x_{1}+\beta}\right)^{-a}}$

We can obtain $\hat{\lambda}, \hat{\theta}, \hat{\beta}$ and $\hat{\alpha}$ through numerical method and the fisher information matrix of Newton Raphson can be used to test for goodness of fit EELD over ELD. The numerical implementation of this hybrid distribution will be done in our subsequent research. 


\section{Conclusions}

By compounding two or more probability distributions, we get the corresponding hybrid distribution with increased number of parameters which is believed to give the newly compounded distribution more flexibility, consistency, stability, sufficiency, uniqueness and wider applicability as compare to its parent distribution. Therefore, Exponentiated-Exponential-Lomax distribution is said to have vast applicability in modeling statistical behavior of stochastic processes such as the growth of tumors' cells in an oncology's study of benign transmogrifying to malignant tumor, studying the consumers' buying behavior and in complex epidemiological studies because of its increased number of parameters which give the hybrid distribution more flexibility to model many stochastic phenomena. The hybrid distribution normally has different patterns or shapes when fitted to simulated data that has well established distribution. It can be used to model data with highly skewed distribution, bimodal or multimodal density. For this reason, it tends to captures non normal data reasonably well; it can be used to model heavily skewed distribution.

\section{References}

[1] Alzaatreh, A. Lee, C.and Famoye, F., (2013).A new method for generating families of continuous distributions. Metron, 71(1), 63-79.

[2] Akinsete,A., Famoye,F., and Lee,C.,(2008). The beta- pareto distribution, Statistics 42(6), 547-563.

[3] Akomolafe A.A and Maradesa A., (2017). Beta-halfnormal Distribution and Its Properties, International journal of Advance Research and Publication vol 1 issued 4 (17-22).

[4] Akomolafe A.A, (2011). Analysis of consumer depth of repeat purchasing pattern: an exploratory study of beverages buying behavior data, Journal of Business and Organizational Development, vol 3 (1-8)

[5] Ashour S.K and Eltehiwy M.A., (2013) . Transmuted Lomax distribution, America Journal of Applied Mathematics and Statistics vol1, 6:121-127.

[6] Badmus, N.I., Ikegwu,M. and Emmanuel,(2013). The Beta-Weighted Weibull Distribution: Some properties and application to Bladder Cancer Data, Applied and Computational Mathematics 2:5.

[7] Barreto-Souza,W., Cordeiro G.M., and Simas A.B., (2011).Some results for beta Frechet distribution. Communications in Stat Statistics Theory \&Methods, 40, 798-811.

[8] Burr, I.W., (1942). Cumulative frequency functions. Annals of Mathematical Statistics, 13, 215-232.

[9] Cordeiro G.M., and de Castro,M.,(2011). A new family of generalized distributions. Journal of Statistical Computation and Simulation 81(7), 883-898.

[10] Cordeiro,G.M., Simas, A.B. and Stosic, B.D., (2011). Closed form expressions for moments of the beta Weibull Distribution, Annals of the Brazilian Academy of Sciences, 8 83, 357-373. 
[11] Cordeiro,G.M., Alexandra, C., Ortega,J.M., Edwin, M.M., and Sarabia, J.M., (2011). Generated distribution ICMA centre. Discusion paper in Finance 1-29.

[12] Eugene, N., Lee,C, and Famoye,F., (2002): Beta-normal distribution and its applications. Communications in Statistic: Theory and methods, 31(4), 497-512.

[13] El-Bassiouny, A.H., Abdo, N.F., Shahen, H.S., (2015). Exponential Lomax Distribution, International Journal of Computer Application volume 121(13), 24-29.

[14] Famoye,F.,Lee, C. and Olumolade,O., (2005). The Beta-Weibull distribution. Journal of Statistical Theory, 4,121-136

[15] Famoye,F., Lee,C. and Eugene, N., (2004). Betanormal distribution: bimodality properties and application. Journal of Modern Applied Statistical Methods, 3, 85-103.

[16] Jones,M.C., (2004). Families of distribution arinsing from distribution of order statistic test 13(1-43)

[17] Kareema A.K. and Mohammed A.B., (2013). Exponential Pareto Distribution, Journal of Mathematical theory and modeling, vol 3, no 5, pp(135-146)

[18] Merovci F. and Puka L.,(2014). Transmuted Pareto Distribution, Probstat Forum vol7, (1-11).

[19] Mahmoud M.R. and Mandouh R.M., (2013). Transmuted frechet, Journal of Applied Science Research vol9 no $10 \mathrm{pp}(555-561)$

[20] Marcelo B., Indranil G., and Cordeiro G.M., (2016), General results for the transmuted Family of Distributions and New Models, Journal of Probability and Statistics.

[21] Mohammad D., and Muhammad A., (2014), on the Mixture of BurrXII and Weibull Distribution, Journal of Statistics Application and Probability, J.Sta.Appl.Pro. 3, No 2, 251-267.

[22] Nadarajah, S., and Kotz, S., (2006). "The exponentiated type distribution',Acta Applicandae Mathematica, vol 92, no2, pp(97-111).

[23] Naradarajah, s., (2006). "The exponential Gumbel distribution with climate application, "Environmetrics vol 17, no $1, \mathrm{pp}(13-23)$.

[24] Okorie E., Akpanta A.C., and Ohakwe J., (2016). The exponential Gumbel Type 2 Distribution: Properties and Application, international Journal of Mathematics and Mathematical Science Volume (2016), Article ID 5898356, 10 pages http://dx.doi.org/10.1155/2016/5898356

[25] Shittu,O.I., and Adepoju, A.K., (2013). On the Beta-Nakagami Distribution: Progress in Applied Mathematics 5:49-5.

[26] Shaw W. and Buckley I. (2007). The Alchemy of probability Distributions:beyond Gram-Charlier Expansions, and a skew-kurtotic-normal distribution from a rank transmutationmap,'Research Report, 2007. 